\title{
AVALIAÇÃO DE DIFERENTES VAZÕES DE AR POR UNIDADE DE VOLUME EM BIORREATORES HETEROTRÓFICOS MICROALGAIS APLICADOS AO TRATAMENTO DE EFLUENTES AGROINDUSTRIAIS
}

\author{
G. J. G. TEIXEIRA ${ }^{1}$, M. M. MARONEZE ${ }^{1}$, A. M. dos SANTOS $^{1}$, L. Q. ZEPKA ${ }^{1}$ e E. \\ JACOB-LOPES ${ }^{1}$
}

${ }^{1}$ Universidade Federal de Santa Maria, Departamento de Tecnologia e Ciência de Alimentos E-mail para contato: jacoblopes@pq.cnpq.br

\begin{abstract}
RESUMO - A fase de cultura de um bioprocesso de microalgas tem a maior carga de energia, e uma parte significativa do consumo de energia está na aeração. Para a produção de bioprodutos a partir de microalgas, é essencial reduzir os requisitos de energia. $\mathrm{O}$ objetivo do trabalho foi avaliar a necessidades de aeração em biorreatores heterotróficos microalgais no tratamento de efluentes do abate e processamento de aves e suínos. Os experimentos foram realizados em biorreatores de colunas de bolhas, alimentados com 1,5L de efluente, 100mg. $\mathrm{L}^{-1}$ de inóculo, $\mathrm{pH}$ ajustado em 7,5 , reator isotérmico operando a $25^{\circ} \mathrm{C}$, aeração continua de 0,5, 1,0, 1,5 VVM e ausência de luminosidade. Os resultados obtidos demonstraram que a vazão por unidade de volume 1,5 VVM apresentou ligeiros ganhos de desempenho, porém a aeração de $0,5 \mathrm{VVM}$ pode ser considerada como o equilíbrio para a operação do biorreator por ter um requerimento de potência menor.
\end{abstract}

\section{INTRODUÇÃO}

O alto consumo de água por frigoríficos resulta em grandes volumes de águas residuárias, sendo que, cerca de 80 a $95 \%$ da água consumida é descarregada como efluente líquido. Estas águas residuárias caracterizam-se principalmente por grandes concentrações de carga orgânica, conteúdo de gordura, nitrogênio, fósforo e sais (Pacheco, 2006).

Por outro lado, estes resíduos agroindustriais apresentam características adequadas para suportar cultivos heterotróficos microalgais, uma vez que, geralmente apresentam razões carbono/nitrogênio e nitrogênio/fósforo adequadas. Além disso, não há a presença de compostos tóxicos ou inibidores de crescimento (Queiroz et al., 2013).

A utilização de microalgas no tratamento de águas residuais pode oferecer uma alternativa menos onerosa e mais eficiente, comparada às formas convencionais de tratamento, como por exemplo, lodos ativados, reatores aeróbios e anaeróbios (Ruiz et al., 2011; Mata et al., 2012). O metabolismo heterotrófico microalgal é fundamentado no consumo de moléculas orgânicas exógenas e nutrientes no escuro, o que promove a remoção de matéria orgânica, nitrogênio e fósforo em uma única etapa. Paralelamente, os poluentes são convertidos em uma biomassa com diversas possibilidades de aplicação, tais como, na 
alimentação humana, produção de energia química, extração de pigmentos, entre outras substâncias celulares de interesse industrial (Queiroz et al., 2007; Lacerda et al., 2011).

Entretanto, a utilização de microalgas para tratamento de efluentes agroindustriais não é isenta de problemas, e análises devem ser feitas para o aperfeiçoamento destes sistemas. A aeração está relacionada com uma considerável parte do consumo de energia, e a maior carga de energia está na fase de cultura de um bioprocesso de microalgas. A redução dos requisitos de energia é vital para condução de processos baseados em microalgas, tornando sistemas energia-positivo (Jones \& Harrison, 2014).

Em face disto, o objetivo deste trabalho foi avaliar as necessidades de aeração do biorreator heterotrófico microalgal, com base em diferentes vazões de ar associadas à resposta metabólica da Phormidium autumnale e consequentemente nos parâmetros cinéticos do bioprocesso.

\section{MATERIAL E MÉTODOS}

\subsection{Microorganismo}

A microalga utilizada foi a Phormidium autumnale obtida a partir de um isolamento de uma área localizada no deserto de Cuarto Cienégas $\left(26^{\circ} 59^{\prime} \mathrm{N}, 102^{\circ} 03^{\prime} \mathrm{W}\right.$ - México). A cultura estoque foi mantida e propagada em agar inclinado, nas condições de $20^{\circ} \mathrm{C}$ e $1 \mathrm{klux}$. $\mathrm{O}$ meio de cultura utilizado foi o BGN (Rippka et al., 1979) adicionado de agar-agar na concentração de $15 \mathrm{~g} \cdot \mathrm{L}^{-1}$.

\subsection{Efluente}

O efluente proveniente do abate e processamento de aves e suínos foi coletado após o tratamento primário (peneira rotativa e equalizador), em uma estação de tratamento de efluentes em Santa Catarina - BR, e congelado até sua utilização. As características iniciais do efluente são: $\mathrm{pH} 5,9$, concentração de matéria orgânica, nitrogênio total e fósforo total de 4099,3 mg.L. ${ }^{-1}, 138,7$ mg. $\mathrm{L}^{-1}$ e 2,84 mg.L $\mathrm{L}^{-1}$, respectivamente.

\subsection{Biorreator}

O aparato experimental foi constituído de um biorreator do tipo coluna de bolhas, construído em vidro de $0,5 \mathrm{~mm}$ de espessura, diâmetro interno de $15 \mathrm{~cm}$, altura de $20 \mathrm{~cm}$ e 3,5 $\mathrm{L}$ de volume de trabalho. O sistema de dispersão de gases do reator constituiu em um difusor de ar de 1,5 cm localizado no centro da base da coluna.

\subsection{Dados Experimentais}

$\mathrm{O}$ experimentos foi operado em batelada, alimentado com 1,5 L de efluente, com $\mathrm{pH}$ ajustado para 7,5, $100 \mathrm{mg} . \mathrm{L}^{-1}$ do inoculo da microalga Phormidium autumnale, temperatura de $25^{\circ} \mathrm{C}$, ausência de luminosidade, e $\mathrm{Q} / \mathrm{V}$ (vazão por unidade de volume) de $0,5,1,0,1,5$ VVM (volume de ar por volume de efluente por minuto).

\subsection{Métodos Analíticos}


As amostras foram coletadas em intervalos regulares de $12 \mathrm{~h}$ e caracterizadas quanto a demanda química de oxigênio (DQO), nitrogênio total (NTK), fósforo total $\left(\mathrm{PO}_{4}{ }^{-3}\right)$ e concentração celular. A demanda química de oxigênio, fósforo total e a concentração de nitrogênio total do efluente foram determinados conforme a metodologia descrita no Standard Methods for the Examinationof Waterand Wastewater (APHA, 2005). A concentração celular foi determinada gravimetricamente por meio da filtração de um volume conhecido de efluente em filtro de $0,45 \mu \mathrm{m}$ de diâmetro. Os testes foram realizados em duplicata e os dados cinéticos referentes à média de quatro repetições.

\subsection{Parâmetros Cinéticos}

Os dados de crescimento celular e consumo de substrato foram utilizados para calcular os seguintes parâmetros cinéticos: produtividade celular $\left(\mathrm{P}_{\mathrm{x}}=\mu_{\max } . \mathrm{X}, \mathrm{mg} / \mathrm{L}\right)$, taxas de consumo de DQO, NTK e $\mathrm{PO}_{4}{ }^{-3}\left(\mathrm{r}_{\mathrm{c}}=\mathrm{dC} / \mathrm{dt}, \mathrm{mg} / \mathrm{L} . \mathrm{h}\right)$ e eficiência de remoção de DQO, NTK e $\mathrm{PO}_{4}{ }^{-3}\left(\mathrm{ER}=\mathrm{C}-\mathrm{C}_{0} / \mathrm{C}_{0}\right)$, onde $\mathrm{X}$ é a densidade celular no tempo $\mathrm{t}=\mathrm{t}(\mathrm{mg} / \mathrm{L})$, t é tempo $(\mathrm{h}), \mathrm{r}_{\mathrm{C}}$ é a taxa de consumo de DQO, NTK e $\mathrm{PO}_{4}^{-3}(\mathrm{mg} / \mathrm{L})$, C é a concentração final de DQO, NTK e $\mathrm{PO}_{4}^{-3}(\mathrm{mg} / \mathrm{L})$ e $\mathrm{C}_{0}$ é a concentração inicial de DQO, NTK e $\mathrm{PO}_{4}^{-3}(\mathrm{mg} / \mathrm{L})$.

\section{RESULTADOS E DISCUSSÃO}

A aeração é um fator determinante no cultivo heterotrófico de microalgas, uma vez que a oxigenação e a mistura interferem significativamente no funcionamento dos sistemas. (Shuler \& Kargi, 2001, Tchobanoglous et al., 2003). Desta forma, os parâmetros cinéticos do biorreator heterotrófico nas condições avaliadas são apresentados na Tabela 1.

Tabela 1 - Parâmetros cinéticos do biorreator heterotrófico a partir do efluente agroindustrial.

\begin{tabular}{cccc}
\hline \multirow{2}{*}{ Parâmetro } & \multicolumn{3}{c}{ Vazão por unidade de volume } \\
\cline { 2 - 4 } & $0,5 \mathrm{VVM}$ & $1,0 \mathrm{VVM}$ & $1,5 \mathrm{VVM}$ \\
\hline $\mathrm{r}_{\mathrm{DQO}}\left(\mathrm{mg} \cdot \mathrm{L}^{-1} \cdot \mathrm{h}^{-1}\right)$ & $46,5^{\mathrm{a}}$ & $46,5^{\mathrm{a}}$ & $46,8^{\mathrm{a}}$ \\
$\mathrm{r}_{\mathrm{N}-\mathrm{NTK}}\left(\mathrm{mg} \cdot \mathrm{L}^{-1} \cdot \mathrm{h}^{-1}\right)$ & $1,65^{\mathrm{a}}$ & $1,74^{\mathrm{a}}$ & $2,3^{\mathrm{b}}$ \\
$\mathrm{r}_{\mathrm{P}-\mathrm{PO}}{ }^{-3}\left(\mathrm{mg} \cdot \mathrm{L}^{-1} \cdot \mathrm{h}^{-1}\right)$ & $0,03^{\mathrm{a}}$ & $0,03^{\mathrm{a}}$ & $0,10^{\mathrm{b}}$ \\
ER $_{\text {DQO }}(\%)$ & $98,5^{\mathrm{a}}$ & $98,5^{\mathrm{a}}$ & $98,5^{\mathrm{a}}$ \\
ER $_{\text {N-NTK }}(\%)$ & $85,6^{\mathrm{a}}$ & $85,6^{\mathrm{a}}$ & $85,6^{\mathrm{a}}$ \\
ER $_{\text {P-PO4 }}$ (3) & $91,3^{\mathrm{a}}$ & $91,3^{\mathrm{a}}$ & $91,3^{\mathrm{a}}$ \\
TDH $(\mathrm{h})$ & $66^{\mathrm{a}}$ & $66^{\mathrm{a}}$ & $48^{\mathrm{b}}$ \\
\hline
\end{tabular}

rc: taxa de consumo de DQO, N-NTK e P-PO ${ }_{4}^{-3}$; ER: eficiência de remoção de DQO, N-NTK e P-PO ${ }_{4}^{-3}$.* letras diferentes em uma mesma linha indicam diferenças significativas pelo teste de Tukey $(\mathrm{p}<0,05)$

A análise dos resultados demonstra que vazões de ar por unidade de volume de 1,5VVM proporcionam um desempenho significativamente superior ao bioprocesso, alcançando-se taxas de consumo de NTK $2,3 \mathrm{mg} \cdot \mathrm{L}^{-1} \cdot \mathrm{h}^{-1}$ e PO4-3 $0,1 \mathrm{mg} \cdot \mathrm{L}^{-1} \cdot \mathrm{h}^{-1}$. Em termos de tempo de detenção hidráulica uma significativa redução foi constatada em vazões por unidade de volume de 1,5 VVM. Adicionalmente eficiências de remoção de 98,5, 85,6 e 91,3 para DQO, NTK e PO4-3 ${ }^{-3}$, respectivamente, foram iguais para todos os processos. 
A Figura 1 apresenta as dinâmicas de crescimento celular e consumo de substrato em diferentes vazões por unidade de volume de ar no biorreator heterotrófico com Phormidium autumnale. A análise dos perfis demonstra uma similaridade bastante pronunciada nas condições de 0,5 e 1VVM, o que inclui dinâmicas sobrepostas de concentração, como no caso do consumo de fósforo total,. Adicionalmente, constatou-se que em termos de consumo de substrato, independente da vazão por unidade de volume utilizada, as concentrações finais atingiram valores idênticos, refletindo como consequência, eficiências de remoção equivalentes. Por outro lado, em termos de crescimento celular, foi observado um comportamento distinto, uma vez que em vazões de 1,5VVM, houve um melhor desempenho na produção de biomassa por Phormidium sp. a partir do efluente agroindustrial. Este melhor desempenho pode estar relacionado a uma maior homogeneidade do biorreator, uma vez que a mistura é favorecida pela maior turbulência no escoamento (JACOB-LOPES et al., 2009). Desta forma, vazões por unidade de volume $(\mathrm{Q} / \mathrm{V})$ de $0,5 \mathrm{VVM}$ serão consideradas como a condição de equilíbrio para a operação do biorreator heterotrófico, uma vez que resultará em menores requerimentos de potência para a operação do processo.
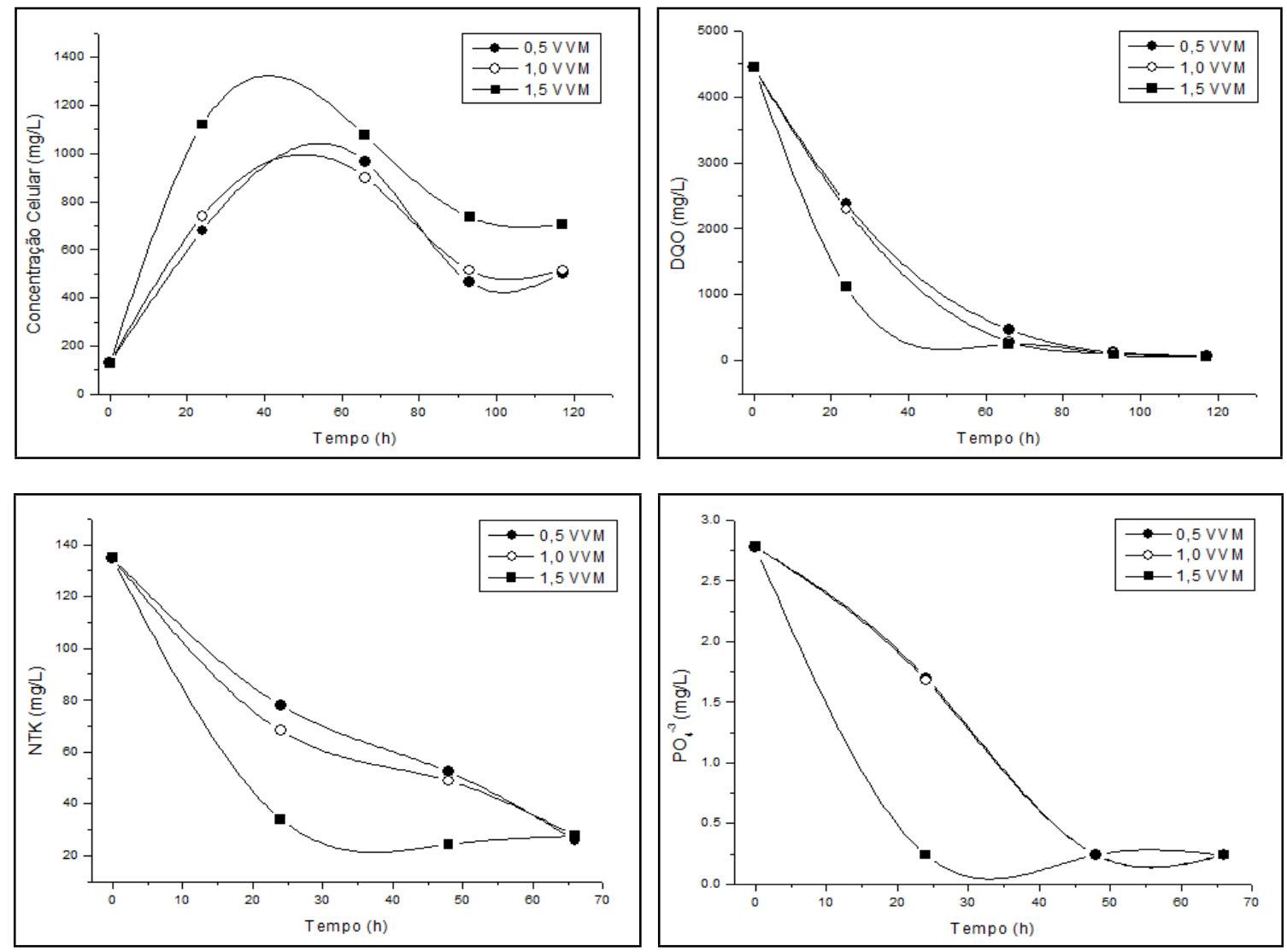

Figura 1 - Dinâmicas de crescimento celular e consumo de substrato em diferentes vazões por unidade de volume de ar $(\mathrm{Q} / \mathrm{V})$ no biorreator heterotrófico, a partir do efluente da indústria processadora de aves e suínos.

\section{CONCLUSÃO}


A operação do biorreator demonstrou que vazões por unidade de volume de 0,5VVM podem ser consideradas como o ponto de equilíbrio entre desempenho cinético e os requerimentos de potência do sistema.

\section{REFERÊNCIAS}

AMERICAN PUBLIC HEALTH ASSOCIATION- APHA. Standard Methods for the Examination of Water and Wastewater. 20. ed. Washington: APHA, 2005.

CONSELHO NACIONAL DO MEIO AMBIENTE- CONAMA RESOLUÇÃO No 357, DE 17 DE MARÇO DE 2005 Publicada no DOU no 053, de 18/03/2005, págs. 58-63.

JACOB-LOPES, E.; REVAH, S.; SHIRAI, K.; HERNANDEZ, S.; FRANCO, T.T. Development of operational strategies to remove carbon dioxide in photobioreactors. Chem Eng J. v.153, p.120-132, 2009.

JONES S.M.J., HARRISON S.T.L. Aeration energy requirements for lipid production by Scenedesmus sp. in airlift bioreactors. Algal Research, v.5, p.249-257, 2014.

LACERDA, L.M.C.; QUEIROZ, I.M.; FURLAN, T. L.; LAURO, M. J.; MODENSI, K.; JACOB-LOPES, E.; FRANCO, T.T. Improvingrefinerywastewater for microalgalbiomassproductionand $\mathrm{CO} 2$ biofixation: Predictivemodelingandsimulation. JournalofPetroleum Science andEngineeringv.78 (2011) p.679-686.

MATA, T.M.; MELO, A.C.; SIMÕES, M.; CAETANO, N.S. Parametric study a brewery efluent treatment by microalgae Scnedesmus obliquus. Bioresource Technology, v.107, p.151-158, 2012.

PACHECO, J. W.; YAMANKA, H. T. Guia técnico ambiental de abates (bovino e suíno). São Paulo: CETESB, 2006. 98p.

QUEIROZ, M.I.; HORNES, M.; MANETTI, A.G.S.; ZEPKA, L.Q.; JACOB-LOPES, E. Fish processing wastewater as a platform of the microalgalbiorefineries. Biosystems Engineering, In Press, 2013.

QUEIROZ, M.I.; JACOB-LOPES, E.; ZEPKA, L.Q.; BASTOS, R.; and GOLDBECK, R. The kinetics of the removal of nitrogen and organic matterfrom parboiled rice effluent by cyanobacteria in a stirred batchreactor.Bioresource Technol., 98(11) (2007) 21632169.

RIPPKA, R.; DERUELLES, J.; WATERBURY, J.B.; HERDMAN, M.; STANIER, R.Y. 1979. Generic assignments strain histories and properties of pure cultures of cyanobacteria.Journal of General Microbiology 111, 1-61.

RUIZ, J.; ÁlVAREZ, P.; ARBIB, Z.; GARRIDO, C.; BARRAGÁN, J.; PERALES, J.A. Effect of nitrogen and phosphorus concentration on their removal kinetic in treated urban wastewater by Chlorella vulgaris. International Journal of Phytoremediation, v. 13, p. 884-896, 2011.

SHULER, M.L.; KARFI, F. Bioprocess Engineering: Basic Concepts. Prentice Hall PTR; 2th edition, 2001.

TCHOBANOGLOUS, G.; BURTON, F.L.; STENSEL, D.Wastewater engineering, treatment and reuse. 4th edition, McGraw-Hill, 2003. 